\title{
Las imágenes inadecuadas de ciencia y de científico como foco de la naturaleza de la ciencia: estado del arte y cuestiones pendientes
}

\author{
Inadequate images of science and scientists as a focus \\ of the nature of science: state of the art and outstanding issues
}

\author{
Alejandro Patricio Pujalte ${ }^{1}$. Leonor Bonan ${ }^{1}$. Silvia Porro ${ }^{2}$. \\ Agustín Adúriz-Bravo ${ }^{1}$
}

\begin{abstract}
Resumen: La presente reseña pretende dar cuenta del estado del arte en la línea de investigación didáctica denominada naturaleza de la ciencia, en particular en lo referente a las imágenes de ciencia y de científico sustentadas por diferentes audiencias. Entre los insumos tenidos en cuenta para esta elaboración se cuenta con las aportaciones surgidas de un curso de posgrado, en el sentido de haber permitido la apropiación de herramientas metadisciplinares destinadas a la redefinición y redimensionamiento del objeto de estudio en el proceso de investigación doctoral. La intención del proyecto es indagar cómo las ideas del profesorado de ciencias acerca de la ciencia y su enseñanza pueden ser obstáculos a la hora de conseguir buenos aprendizajes en estudiantes que acceden a la escuela con desventaja en términos de capital cultural. En este sentido, cobra importancia el grado de profundidad en la comprensión de la naturaleza de la ciencia que posee el profesorado.
\end{abstract}

Palabras clave: Enseñanza de la ciencia. Formación del profesorado. Naturaleza de la ciencia. Educación científica.

\begin{abstract}
This review aims to describe the state of the art in research into the nature of science, particularly in relation to images of science and scientists provided for different audiences. Among the inputs that were considered for this work, we took into account those that emerged from a postgraduate course. This allowed the use of meta-disciplinary tools, aimed at redefining and reshaping the object of study in the Ph.D. research process. The intention of the project is to investigate how the ideas of science teachers about science and its teaching can be obstacles to achieving good learning in students entering school which puts them at a disadvantage in terms of cultural capital. In this sense, the degree of depth in understanding the nature of science held by teachers becomes important.
\end{abstract}

Keywords: Science teaching. Teacher education. Nature of science. Science education.

\footnotetext{
${ }^{1}$ Centro de Formación e Investigación en Enseñanza de las Ciencias, Facultad de Ciencias Exactas y Naturales, Universidad de Buenos Aires, Intendente Güiraldes, 2160, Ciudad Universitaria, C1428EGA, Buenos Aires, Argentina. E-mail: <ap_pujalte@yahoo.com.ar>

${ }^{2}$ Grupo de Investigación en Enseñanza de las Ciencias (GIECIEN), Universidad Nacional de Quilmes, Buenos Aires, Argentina.
} 


\section{Introducción}

Cuando se les pregunta a las y los estudiantes cómo es que se imaginan a una persona que trabaja en ciencias y se les pide que la dibujen en su ambiente de trabajo en un día típico, los resultados suelen ser similares. En la mayoría de los casos dibujan científicos varones, con lentes y guardapolvo, a menudo calvos o con el pelo revuelto, trabajando solos en un lugar que suele ser un laboratorio, con características semejantes a las de un laboratorio escolar (CHAMBERS, 1983; MEAD; METRAUX, 1957). Las indagaciones realizadas en este sentido muestran una recurrencia a estos clisés en diferentes niveles educativos y en distintas culturas. Es frecuente que a estas indagaciones a través de solicitar el dibujo, también se las acompañe con preguntas que apuntan a que las y los estudiantes expliciten por escrito algunas características. Las descripciones que se obtienen por este medio también suelen ser muy coincidentes: este científico típico es distraído, absorbido por su trabajo, con poca vida social, ocupado en cosas que sólo él puede entender, sin familia o amigos, sin otros intereses o motivaciones. Todos estos rasgos hasta aquí señalados y algunos otros corresponden a un estereotipo. Se afirma que esa imagen de científico que se plasma en los dibujos es un epifenómeno de una particular imagen de ciencia, en el sentido de que la gente personifica y pone en el estereotipo de científico que dibuja su propia imagen de ciencia ${ }^{3}$.

El presente trabajo se inscribe en una de las líneas de investigación que el Grupo de Epistemología, Historia y Didáctica de las Ciencias Naturales (GEHyD) viene llevando adelante desde hace algunos años (ADÚRIZ-BRAVO et al., 2006) y surge como producción final del curso de posgrado "Tópicos de la Didáctica de las Ciencias Naturales" (a cargo de la segunda autora de este artículo), como instancia de redefinición y redimensionamiento del objeto de estudio en el proceso de investigación doctoral del primer autor (dirigido y codirigido por los dos últimos autores, respectivamente). Es así que intentamos realizar aquí un recorrido por lo hecho hasta el momento en materia de las indagaciones a las que venimos haciendo referencia, sin pretensiones de exhaustividad. Se quiere presentar un panorama general del estado del arte en torno a la cuestión de las imágenes de ciencia y de científico en diversas poblaciones y las relaciones que éstas tienen con el logro de una educación científica de calidad para todas y todos. El trabajo finaliza con algunas conclusiones siempre provisorias y abriendo el campo a una serie de preguntas que nos parece pertinente tener en cuenta para futuras investigaciones.

A modo de hoja de ruta, anticipamos a continuación los aspectos que se abordarán a lo largo del trabajo:

1. Se caracteriza la línea de investigación en didáctica denominada naturaleza de la ciencia como marco de las indagaciones que son objeto de nuestro estudio.

2. Se proponen algunos elementos teóricos para tratar de definir los alcances de lo que llamaremos imagen de ciencia y de científico a lo largo del trabajo.

3. Se realiza una reseña de algunas de las investigaciones acerca de la imagen de ciencia y científico en estudiantes, prestando especial atención a los resultados, a las variantes metodológicas y a los factores que intervienen en la constitución de las imágenes.

${ }_{3}$ Reportaje a Adúriz-Bravo. (STEKOLSCHIK, 2008). 
4. Se centra la mirada en el profesorado como factor de especial relevancia y en la caracterización de la propia imagen de ciencia de las y los profesores.

5. Finalmente, y a modo de conclusión, se plantean algunos interrogantes que invitan a profundizar en el estudio de las relaciones entre las concepciones sobre la naturaleza de la ciencia del profesorado y la calidad de la enseñanza impartida, de acuerdo a las finalidades de la educación científica que se presuponen para estudiantes provenientes de contextos socioeconómicamente desfavorecidos.

\section{Desarrollo}

\section{La naturaleza de la ciencia}

Estas indagaciones se enmarcan en la línea de investigación en didáctica de las ciencias denominada naturaleza de la ciencia (NOS, por su sigla en inglés) que en los últimos veinte años viene examinando tanto las imágenes de ciencia de estudiantes como las de profesoras y profesores de ciencias.

En realidad, este interés de la línea NOS en explorar estas imágenes surge de su principal preocupación: la alfabetización científica de calidad para todos y todas a partir de la reflexión curricular que plantea la necesidad de que la población no sólo deba saber ciencias sino saber sobre ciencias. Esto es, que además de adquirir los contenidos científicos, la ciudadanía pueda responderse las preguntas acerca de qué es la ciencia, cómo cambia en el tiempo y cómo se relaciona con la sociedad y la cultura (ADÚRIZ-BRAVO, 2009). Esto implica una incorporación de contenidos que incluyan una reflexión crítica sobre las ciencias naturales, y, en tanto reflexión, de carácter metadiscursivo. Estos contenidos: “[...] de alguna forma se desprenden de las metaciencias (epistemología, historia y sociología de la ciencia) y pretenden generar en los ciudadanos y ciudadanas imágenes de ciencia más ajustadas a lo que actualmente se sabe sobre el conocimiento y la actividad científicas" (ADÚRIZ-BRAVO, 2005, p. 24, cursivas en el original).

Lo que muestran los estudios exploratorios es que en general las poblaciones estudiadas no cuentan con visiones adecuadas de la naturaleza de la ciencia. Mucho se ha escrito acerca de la importancia de la incorporación de las metaciencias a la educación científica. Driver et al. (1996) proponen ciertos argumentos o razones a la hora de defender la inclusión de naturaleza de la ciencia en la enseñanza de las ciencias:

Razón utilitaria: Para dar sentido a la ciencia y para poder manejarse con los objetos y los procesos tecnológicos.

Razón democrática: Para la toma de decisiones informada en las cuestiones sociocientíficas.

Razón cultural: Para apreciar el valor de la ciencia como parte de la cultura contemporánea.

Razón moral: Ayuda a desarrollar la comprensión de las normas de la comunidad científica que involucran compromisos morales de valor universal para la sociedad.

Aprendizaje de las ciencias: Comprender la naturaleza de la ciencia facilita el aprendizaje de los contenidos de las asignaturas científicas.

Siguiendo a Lederman (2006) se puede decir que la investigación en la línea NOS se ha centrado progresivamente en los siguientes focos de atención: 
a) Concepciones de las y los estudiantes

b) Curriculum

c) Concepciones de las y los profesores

d) Propuestas para la mejora de las concepciones del profesorado

e) La relativa eficacia de diversas prácticas instruccionales

Llegados a este punto, Lederman (2006) se atreve a realizar algunas generalizaciones a la luz de los numerosos estudios realizados a la fecha:

a) Las y los estudiantes de secundaria no suelen tener "adecuadas" concepciones acerca de la naturaleza de la ciencia.

b) Las y los profesores de secundaria no suelen tener "adecuadas" concepciones acerca de la naturaleza de la ciencia.

c) Se aprenden mejor las concepciones acerca de la naturaleza de la ciencia mediante la enseñanza explícita y reflexiva antes que implícitamente a través de experiencias donde simplemente "se hace" ciencia.

d) Las concepciones de las y los profesores acerca de la naturaleza de la ciencia no se trasladan automáticamente y necesariamente a la práctica de aula.

e) El profesorado no considera a la naturaleza de la ciencia como un objetivo de enseñanza de igual estatus con respecto a los "tradicionales" objetivos de las asignaturas científicas.

Con todo, respecto de las instancias de formación inicial del profesorado en aspectos NOS, se señala que necesariamente deben tener en cuenta las imágenes o visiones globales acerca de la ciencia que traen consigo los futuros profesores y profesoras, en el sentido de que pueden constituirse en verdaderos obstáculos a la hora de lograr aprendizajes genuinos de adecuadas nociones acerca de la naturaleza de la ciencia (ABD-EL-KHALICK, 2001).

\section{Acerca de las imágenes}

La fuerte impronta del estereotipo del científico en el imaginario colectivo parecería indicar un origen común, habida cuenta de su extensión, atravesando fronteras, etnias, diferencias etarias, de género y de nivel educativo. Incluso se evidencia en las propias científicas y científicos y desde ya en profesoras y profesores de ciencias de todos los niveles. Se suelen citar diversas fuentes de estas imágenes distorsivas: los cómics (GALLEGO TORRES, 2007) los dibujos animados (VILCHEZ-GONZÁLEZ; PALACIOS, 2006), la literatura (HAYNES, 2003) el cine (WEINGART, 2007), la TV y la publicidad (CAMPANARIO; MOYA; OTERO, 2001). Aunque cabría preguntarse si son el origen o simplemente las refuerzan. La extensa bibliografía a nivel internacional usa diferentes nombres para hablar de este conjunto de características que las indagaciones revelan: imágenes, imaginarios, visiones, concepciones, creencias, representaciones, percepciones. En algunos casos denotan la adscripción a un marco teórico de referencia, como cuando se alude a las representaciones sociales, desde la perspectiva de Moscovici (MORA, 2002). Si bien son reconocibles matices en todas estas denominaciones, creemos que hay un denominador común si se las entiende en sentido amplio. Así, en este trabajo las usaremos indistintamente. 


\section{Indagaciones de la imagen de científico en estudiantes}

Uno de los trabajos pioneros en investigar esta temática es el de Margaret Mead y Rhoda Metraux (1957) indagando sobre una muestra de 35000 estudiantes de secundaria estadounidenses acerca de cómo veían a los científicos. A continuación presentamos dos figuras que sistematizan los rasgos más sobresalientes de los resultados de este estudio (Figuras 1 y 2).

Ha transcurrido bastante tiempo desde esta investigación y no parece que esta imagen haya cambiado significativamente. El estereotipo del científico sigue incólume y no constituye un modelo al cual la mayoría de las y los estudiantes quieran adherir.

Trabajos recientes muestran una persistencia de esa doble representación, positiva y negativa, en el imaginario del estudiantado. De esta manera, "Mientras que la mayoría de la gente expresa respeto y admiración por los científicos, el estereotipo dominante desalienta a quienes no se identifican con él cuando se piensan a sí mismos como científicos" (LESLIE-PELECKY; BUCK; ZABAWA, 2005).

Muchos estudios han revelado la temprana formación de esta imagen; la evidencia muestra que ya a los seis o siete años las niñas y los niños producen estas representaciones (NEWTON; NEWTON, 1998). De hecho, son ampliamente reconocidos los trabajos que resultan de la aplicación del Draw a Scientist Test (DAST) a niñas y niños de los primeros años de la educación primaria, donde se les pide que dibujen a una persona que se dedica a la ciencia

Figura 1. Aspectos recurrentes respecto de la apariencia de las personas que se dedican a la actividad científica y acerca de las características del ambiente donde suelen trabajar

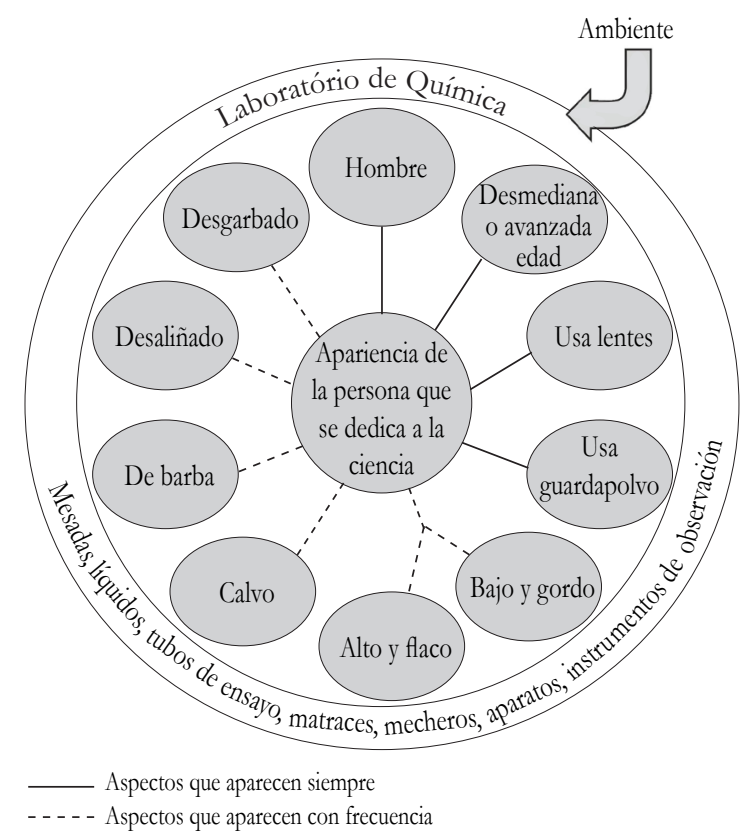

Fuente: Mead y Metraux (1957). 
Figura 2. Aspectos positivos y negativos de la imagen de las personas que se dedican a la ciencia

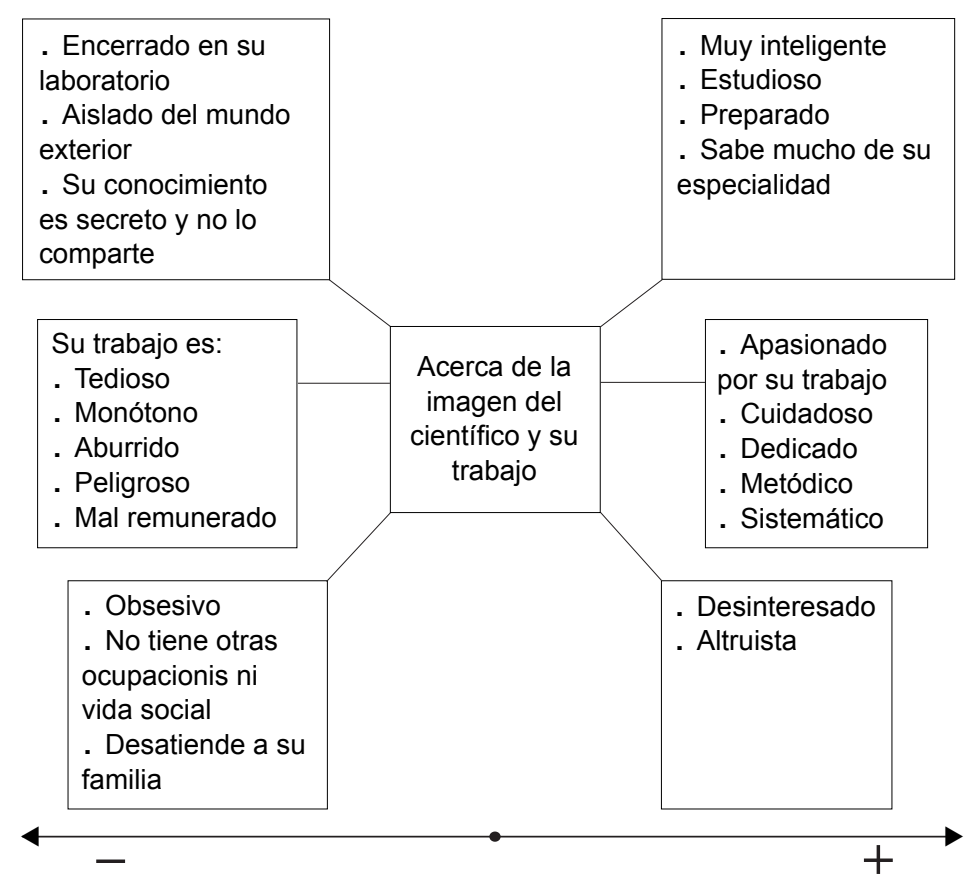

Fuente: Mead y Metraux (1957).

en su ambiente de trabajo (CHAMBERS, 1983). Una de las cuestiones más abordadas en estos análisis es la percepción de género en los dibujos, notándose una amplia recurrencia al científico varón. Sin embargo, existen matices: Se ha señalado que a edades tempranas suele haber una representación del propio género, y, por una cuestión madurativa, las niñas suelen hacer dibujos más fácilmente reconocibles como pertenecientes al género femenino que en el caso de los niños, donde a veces esa distinción no se hace sencilla. Los varones, en general, tienden a encasillar más al científico dentro del estereotipo (LOSH; WILKE; POP, 2008).

Cabe destacar los proyectos de envergadura internacional abocados a indagar qué es lo que sucede con estas percepciones y actitudes de las alumnas y los alumnos al atravesar las fronteras: uno de ellos ha sido "The SAS Study", dirigido por Svein Sjøberg, de la Universidad de Oslo (SJØBERG, 2000). En este estudio las niñas y los niños de los países en vías de desarrollo presentaron una visión sobre la ciencia y la tecnología mucho más positiva que la de aquellas y aquellos de los países ricos. Mientras que en estos últimos países, los niños (principalmente varones) representaban al científico como una persona cruel y loca, en los países en vías de desarrollo los consideraban ídolos y héroes. Se señala que en los países desarrollados existe un problema con la imagen pública de la ciencia y una creciente preocupación por la baja de la matrícula en las carreras relacionadas con la ciencia y la tecnología. Al preguntarse acerca de esta suerte de contradicción -dado que estos niños y jóvenes viven en sociedades basadas en 
el conocimiento científico y tecnológico-, sostienen una explicación posible: que se trata del resultado de una baja comprensión pública de la ciencia, atribuible a una enseñanza de mala calidad, como también de una mala imagen reflejada en los medios.

Entre los materiales publicados que surgen de la investigación de la imagen de científico de las niñas y los niños, se destaca el libro "El científic dibuixat"4 (ESCALAS TRAMULLAS; RUIZ; ZORRILLA, 2009). Se trata de una selección de los 250 dibujos que realizaron más de 300 niñas y niños catalanes, entre 6 y 18 años, como participantes del concurso "Dibuja un científico" en el marco del proyecto Discover the Researchers' Facets que promueve la Comisión Europea. En este concurso, se pedía a los participantes que dibujaran un científico o una científica, sin especificar nada más, a fin de condicionar lo menos posible el resultado. El contenido de los dibujos fue analizado por un grupo de expertos coordinado por M. Teresa Escalas, del Observatorio de la Difusión de la Ciencia (ODC) de la Universidad Autónoma de Barcelona (UAB) y por David Segarra, del portal Investigación en Acción de la Agencia de Gestión de Ayudas Universitarias y de Investigación (AGAUR), con la colaboración de las investigadoras Juanita Zorrilla y Aída Homs. El equipo utilizó el DAST como metodología de análisis. La publicación se constituye como un libro para pensar, descubrir y dibujar, ya que incluye propuestas que buscan que el lector reflexione sobre su propia imagen de científico, a través de varias actividades, y con una propuesta final que incluye un test para estimar qué tan estereotipada es la imagen.

En el ámbito latinoamericano es destacable además el trabajo llevado adelante por el Equipo de Investigación en Enseñanza de las Ciencias Naturales de la Federación Uruguaya de Magisterio: Han hallado evidencia a favor de que los rasgos estereotípicos acerca de la ciencia y los científicos se acentúan con el decurso de la escolaridad. Además, estos estudios parecerían indicar que las niñas y los niños del nivel preescolar e inicial poseen representaciones acerca de la ciencia mucho más ricas y variadas en las que es muy potente la autoidentificación con la figura del científico, en el marco de diversos escenarios posibles para la actividad científica (PUJALTE et al., 2011).

Respecto del DAST y su aplicación, han habido reformulaciones, algunas de ellas a partir de ciertas críticas. Tal es el caso del trabajo llevado adelante por Manzoli et al. (2006), donde se señalan algunas consideraciones respecto de este test en los siguientes sentidos: (a) que no revela cómo y de dónde se construye el estereotipo, (b) que da una imagen estática, no permite que el estudiante dé detalles de la ciencia como proceso, y (c) que no queda del todo claro si es que el DAST mide los estereotipos de las y los estudiantes o bien sucede que los dibujan así para hacerlos reconocibles como tales por quienes le demandan el dibujo (más allá de que ésa sea su "verdadera“ imagen de científico). El estudio de referencia se realizó en Italia, con niñas y niños de 8 y 9 años, donde además de pedírseles el dibujo se les solicitó que inventaran una historia que tuviera al científico como uno de sus protagonistas (otros investigadores en el tema también han echado mano de este recurso. Véase REIS; GALVÃO, 2006). A partir de los resultados, los autores concluyen que, por un lado, las representaciones que las niñas y los niños manifiestan van más allá del estereotipo, que en realidad lo usan como "esqueleto" sobre

\footnotetext{
4 "El científico dibujado".
} 
el cual vuelcan aspectos que tienen más que ver con lo que captan de los medios y, por otro, que revelan sorprendentes niveles de conciencia acerca de los aspectos sociales, éticos y políticos de la actividad científica y de los métodos y prácticas de los científicos.

Con todo, lo que parece quedar claro es que, si bien esta imagen estereotipada se forma tempranamente, a medida que la escolaridad avanza, los rasgos más característicos se acentúan con fuerza, con el correlato correspondiente del desinterés por las asignaturas científicas por parte de las y los jóvenes y la merma consiguiente de matrícula en las carreras científicas. Esta exclusión además tiene una importante componente de género, relegando a las mujeres a un plano muy secundario. En este sentido, Jones, Howe y Rua (2000) advierten que la escuela - a pesar de ser un ambiente que por lejos no está libre de prejuicios de género - debe ser el lugar donde las y los estudiantes puedan encontrar los mejores valores y actitudes acerca de la ciencia. Señalan que las profesoras y los profesores no pueden eludir la responsabilidad de presentar a la ciencia como igualmente apropiada para chicos y chicas, de pretender que también las chicas sean capaces de utilizar las herramientas científicas con facilidad, y de lograr que tanto chicos como chicas se involucren reflexivamente en las actividades científicas. Además concluyen que continuar con el statu quo sin transformar la cultura es condenar a las estudiantes a permanecer en los bordes de la ciencia. En esa misma línea, Wyer (2003) afirma que, respecto a la persistencia y el no abandono de las carreras científicas por parte de las mujeres, existe una estrecha relación con las imágenes positivas de científicos, con las actitudes positivas respecto de la igualdad de género y con las experiencias positivas en las aulas de ciencias. La amplia instalación de los estereotipos que de alguna manera prescriben qué grupos pueden acceder y tener éxito en el ámbito del conocimiento científico, resultan en una desidentificación por parte de los grupos aludidos respecto de la ciencia, que ya dan por sentada su falta de habilidad para ello. Esto es particularmente notorio en el caso de las minorías étnicas y de las mujeres, como bien señala Steele (1997).

\section{En procura del cambio}

Habida cuenta de la situación relatada, han sido desarrollados muchos trabajos de investigación con el objetivo de evaluar el impacto de intervenciones específicas que buscan operar sobre la imagen de científico de las y los estudiantes, para procurar una imagen de ciencia mucho más inclusora. La mayoría de ellos coincide en que las intervenciones son tanto más efectivas cuanto más temprano se hagan, esto es, en los primeros años de la escolaridad primaria, cuando las niñas y los niños tienen las primeras aproximaciones formales a los contenidos científicos. Por ejemplo, Bodzin y Gehringer (2001) estudiaron el efecto de involucrar a científicas y científicos en las secuencias didácticas destinadas a enseñar ciencia a chicas y chicos de escuela primaria. Mediante el uso de pretest y postest pudieron evidenciar en los dibujos cambios significativos en los aspectos más recurrentes del estereotipo del científico. Los autores afirman además que esa no es la única forma de llevar los científicos al aula, sino que existen diversas formas de hacerlo, como por ejemplo a través del correo electrónico, las conferencias virtuales, etc., como también la oportunidad de hacer visitas a los laboratorios y lugares de trabajo.

Cakmakci et al. (2011) sostienen asimismo que tanto los resultados de la investigación empírica sobre la imagen de científico de los estudiantes como también los estudios teórico-analíticos acerca de la naturaleza del conocimiento, el pensamiento, el aprendizaje y la enseñanza, 
pueden usarse para diseñar actividades de enseñanza destinadas a mejorar las ideas de las y los estudiantes acerca de la ciencia y los científicos. Coinciden en señalar que en general estas estrategias no son lo suficientemente utilizadas o bien se aplican muy tarde.

\section{El rol del profesorado y la imagen de ciencia como obstáculo a la hora de enseñar ciencias naturales}

Como hemos señalado hasta acá, las imágenes estereotipadas del científico nos revelan una versión poco feliz respecto de la ciencia que se enseña y que se aprende. La investigación internacional la suele catalogar como visiones deformadas, imágenes distorsionadas o inadecuadas (ADÚRIZ-BRAVO, 2001; CHUNG-CHIH CHEN; TAYLOR; ALDRIDGE, 1997; FERNÁNDEZ et al., 2002; HODSON, 1998; HUGO; ADÚRIZ-BRAVO, 2003; MANASSERO; VÁZQUEZ, 2001). En muchos trabajos se afirma que estas visiones deformadas se transmiten cuando se enseña ciencia (FERNÁNDEZ et al., 2002), de ahí la importancia de conocer la imagen de ciencia que traen las profesoras y los profesores de ciencias, en formación y en servicio, para poder intervenir sobre ella. Se suele afirmar que, en general, el profesorado no es consciente de sus propias representaciones, de ahí la importancia de generar instancias que promuevan su elicitación, para poder ponerlas en cuestión.

En tren de caracterizar esa imagen de ciencia subyacente, las investigaciones referidas en el párrafo anterior (ver, a modo de ejemplo, FERNÁNDEZ et al., 2002) coinciden en que dicha imagen surge de una visión marcadamente empiroinductivista, que considera a la ciencia como construcción ahistórica, marcadamente individualista, independiente de valores, ideologías, intereses y contextos y por tanto, neutral, objetiva y sin dudas infalible y dueña de la verdad. Al mismo tiempo, también hay consenso en que se muestra como una empresa elitista y exclusora, esencialmente masculina, fundada en una racionalidad científica centrada en un único método. Suele acentuarse su carácter críptico y hermético, que sólo puede ser descifrado por verdaderos "iniciados".

La bibliografía internacional es muy abundante en trabajos de investigación sobre las concepciones del profesorado y sobre instancias de intervención que apuntan a promover una adecuada comprensión de la Naturaleza de la Ciencia en el profesorado (ACEVEDO DÍAZ, 2007 a 2007b). En esta dirección se viene remarcando la virtual ineficacia de los cursos aislados sobre naturaleza de la ciencia apostando por la enseñanza explícita y reflexiva, vinculada con los contenidos científicos que las y los futuros docentes deberán enseñar en el aula. (BUARAPHAN, 2009).

Volviendo ahora a las generalizaciones a las que hace referencia Lederman (2006), a las que aludimos en la primera parte de este trabajo, en relación con las investigaciones realizadas en la línea NOS sobre las concepciones de estudiantes y profesorado, cabe señalar que, sobre el cuarto punto por él reseñado: "Las concepciones de las y los profesores acerca de la naturaleza de la ciencia no se trasladan automáticamente y necesariamente a la práctica de aula" (LEDERMAN, 2006, p. 6), no hay consenso al respecto. Otros autores han encontrado coherencia entre la imagen de ciencia sustentada por el profesorado y sus prácticas de aula (CAETANO; NETO, 2005; TSAI, 2007).

Con todo, la bibliografía internacional coincide en que median factores contextuales (experiencia docente, presión por "cumplir el programa", factores institucionales, falta de re- 
cursos...) que hacen que el hecho de que el profesorado posea una adecuada concepción de la NOS es condición necesaria pero no suficiente para que ésta se traslade a las y los estudiantes. Dentro de los factores mencionados, cobra especial importancia el grado de profundidad en la comprensión de la naturaleza de la ciencia que poseen las profesoras y los profesores de ciencias (ABD-EL-KHALICK, 2005).

Como señala Adúriz-Bravo (2007, p. 20):

$[\mathrm{N}]$ os parece central abocarnos a estudiar, desde la didáctica de las ciencias naturales, posibles formas de integrar significativamente la naturaleza de la ciencia en la formación de los profesores de ciencias para todos los niveles educativos. La naturaleza de la ciencia podría cumplir, en esta formación, al menos tres fines distintos: 1. Un fin intrínseco, por el cual ella provee a los profesores una reflexión crítica, con fundamento teórico, sobre la ciencia; 2. Un fin cultural, por el cual los profesores se apropian de un contenido valioso dentro del panorama de creaciones intelectuales humanas; y 3. Un fin instrumental, por el cual la naturaleza de la ciencia ayuda a que los profesores aprendan mejor los contenidos de ciencias y sean luego capaces de enseñarlos más significativamente.

\section{Las concepciones sobre NOS del profesorado y las finalidades de la educación científica: conclusiones provisorias y posibles horizontes}

En el amplio panorama que hemos presentado, se abren algunos interrogantes respecto de los probables vínculos entre las concepciones acerca de la naturaleza de la ciencia y las concepciones psicopedagógicas (cómo enseñar ciencias) y sociales (para qué enseñar ciencias) (PUJALTE; PORRO, 2009). Por un lado, parece existir consenso en que la educación científica para la ciudadanía en el siglo XXI no puede limitarse a la formación propedéutica (ACEVEDO DÍAZ, 2004; ACEVEDO DÍAZ et al., 2005; EL-HANI; MORTIMER, 2007; FOUREZ, 1997; FURIÓ et al., 2001). Sin embargo, cabe preguntarse en qué medida el discurso "democratizante" y "políticamente correcto" se traduce en la práctica en acciones coherentes o por el contrario tiende a compatibilizarse más bien con una mirada de déficit y asistencialista para aquellos estudiantes de contextos social y económicamente desfavorecidos. De la mano con lo anterior, varios autores vienen desarrollando la idea de que existe una correlación ente las concepciones NOS que sustentan las y los docentes y sus visiones acerca de cómo se aprende ciencia y cómo se enseña (GALLEGOS; BONILLA, 2009; TSAI, 2002). Parece haber acuerdo en pensar como sugiere Tsai (2002) - que las creencias de las profesoras y los profesores de ciencia acerca de la enseñanza, el aprendizaje y la propia ciencia, al menos, imponen ciertos efectos sobre las 'percepciones' respecto de la práctica de la enseñanza. Incluso el autor plantea que cambiar las creencias sobre la enseñanza y el aprendizaje de la ciencia puede constituirse en un prerrequisito para lograr cambiar las concepciones acerca de la naturaleza de la ciencia, o viceversa, tal sería el grado de vinculación entre unas y otras. Finalmente, se podría sustentar más aún lo anterior si se piensa que determinado perfil de concepciones o creencias se "activa" en determinados contextos: según Barnett y Hodson (2001), un mismo profesor enseña de forma distinta un mismo contenido a diferentes estudiantes, dependiendo de las condiciones particulares de esos 
estudiantes y de la escuela. La enseñanza sería "contexto-específica” y por ello, las decisiones de los docentes en el aula están determinadas por el contexto, por las condiciones sociales y culturales específicas. Y no siempre esa adaptación al contexto se traduce en una educación científica de calidad, principalmente cuando se trata de aquellos y aquellas estudiantes a los que hacíamos referencia más arriba (GÓMEZ et al., 2004), que acceden a la escuela media con desventaja en términos del capital cultural que la escuela legitima (BOURDIEU; PASSERON, 2003). Consideramos importante una profundización respecto de lo que se sabe acerca de las concepciones NOS del profesorado y sus relaciones con los aspectos que hemos señalado en estas últimas líneas.

\section{Referencias}

ABD-EL-KHALICK, F. Embedding nature of science instruction in preservice elementary courses: abandoning scientism but ... Journal of Science Teacher Education, Dordrecht, v. 12, n. 3, p. 215- 233, 2001.

Developing deeper understandings of nature of science: the impact of a philosophy of science course on preservice science teachers' views and instructional planning. International Journal of Science Education, Abingdon, v. 27, n. 1, p. 15-42, 2005.

ACEVEDO DÍAZ, J. A. Reflexiones sobre las finalidades de la enseñanza de las ciencias: educación científica para la ciudadanía. Revista Eureka sobre Enseñanza y Divulgación de las Ciencias, Cadiz, v. 1, n. 1, p. 3-16, 2004.

. Una selección de artículos sobre decisiones tecnocientíficas y enseñanza de las ciencias (I). Revista Eureka sobre Enseñanza y Divulgación de las Ciencias, Cadiz, v. 4, n. 1, p. 195-201, $2007 \mathrm{a}$.

. Una selección de artículos sobre decisiones tecnocientíficas y enseñanza de las ciencias (II).

Revista Eureka sobre Enseñanza y Divulgación de las Ciencias, Cadiz, v. 4, n. 2, p. 358-363, 2007b.

ACEVEDO DÍAZ, J. A. et al. Naturaleza de la ciencia y educación científica para la participación ciudadana: una revisión crítica. Revista Eureka sobre Enseñanza y Divulgación de las Ciencias, Cadiz, v. 2, n. 2, p. 121-140, 2005.

ADÚRIZ-BRAVO, A. Integración de la epistemología en la formación del profesorado de ciencias. 2001. 622 f. Tesis (Doctorat en Didàctica de les Ciències Experimentals) - Universitat Autònoma de Barcelona, Bellaterra, 2001.

- ¿Qué naturaleza de la ciencia hemos de saber los profesores de ciencias?: una cuestión actual de la investigación didáctica. Tecné, Episteme y Didaxis, Bogotá, p. 23-33, 2005. (Número extraordinario: $2^{\circ}$ Congreso sobre Formación de Profesores de Ciencias).

ADÚRIZ-BRAVO, A. La naturaleza de la ciencia en la formación de los profesores de ciencias naturales. In: GALLEGO, R.; PÉREZ, R.; TORRES, L. (Ed.). Didáctica de las ciencias: aportes para una discusión. Bogotá: Universidad Pedagógica Nacional, 2007. p. 17-36.

ADÚRIZ-BRAVO, A. La naturaleza de la ciencia "ambientada" en la historia de la ciencia.

Enseñanza de las Ciencias, Basrcelona, p. 1178-1181, 2009. (Número Extra: VIII Congreso Internacional sobre Investigación en Didáctica de las Ciencias) 
ADÚRIZ-BRAVO, A. et al. Las imágenes de ciencia y de científico en una propuesta de educación inclusiva para todos y todas. In: EDUCACIÓN social: formación, realidad y retos. Granada: Editorial Universitario, 2006. p. 427-435.

BARNETT, J.; HODSON, D. Pedagogical context knowledge: toward a fuller understanding of what good science teachers know. Science Education, Hoboken, v. 85, n. 4, p. 426-453, 2001.

BODZIN, A.; GEHRINGER, M. Breaking science stereotypes: can meeting actual scientists change students' perceptions of scientists? Science and Children, Arlington, v. 38, n. 4, p. 36-41, 2001.

BOURDIEU, P.; PASSERON, J. Los herederos: los estudiantes y la cultura. Buenos Aires: Siglo XXI, 2003.

BUARAPHAN, K. Preservice and inservice science teachers' responses and reasoning about the nature of science. Educational Research and Reviews, Lagos, v. 4, n. 11, p. 561-581, 2009.

CAETANO, H.; NETO, A. J. Natureza e ensino da ciência: investigando as concepçoes de ciência dos professores. Enseñanza de las Ciencias, Barcelona, p. 1-5, 2005. (Número Extra. VII Congreso Internacional sobre Investigación en Didáctica de las Ciencias).

CAKMAKCI, G. et al. Promoting an inclusive image of scientists, among students: towards research evidence-based practice. International Journal of Science and Mathematics Education, Dordrecht, v. 9, n. 3, p. 627-655, 2011.

CAMPANARIO, J. M.; MOYA, A.; OTERO, J. C. Invocaciones y usos inadecuados de la ciencia en la publicidad. Enseñanza de las Ciencias, Barcelona, v. 19, n. 1, p. 45-56, 2001.

CHAMBERS, D. W. Stereotypic images of the scientist: the draw-a-scientist test. Science Education, Hoboken, v. 67, n. 2, p. 255-265, 1983.

CHUNG-CHIH CHEN, C. C.; TAYLOR, P. C.; ALDRIDGE, J. M. Development of a questionnaire for assessing teachers' beliefs about science and science teaching in Taiwan and Australia. In:

ANNUAL MEETING OF THE NATIONAL ASSOCIATION FOR RESEARCH IN SCIENCE TEACHING, Oak Brook, 1997. Disponible en: < http://files.eric.ed.gov/fulltext/ED405219.pdf >. Visitado el: 18 Jul. 2014.

DRIVER, R. et al. Young people's images of science. Buckingham: Open University Press, 1996.

EL-HANI, C. N.; MORTIMER, E. F. Multicultural education, pragmatism, and the goals of science teaching. Cultural Studies of Science Education, Dordrecht, v. 2, n. 3, p. 657-702, 2007.

ESCALAS TRAMULLAS, M.; RUIZ, I.; ZORRILLA, J. (Ed.). El científic dibuixat. Barcelona: Generalitat de Catalunya, 2009.

FERNÁNDEZ, I. et al. Visiones deformadas de la ciencia transmitidas por la enseñanza. Enseñanza de las Ciencias, Barcelona, v. 20, n. 3, p. 477-488, 2002.

FOUREZ, G. (Ed.). Alfabetización científica y tecnológica: acerca de las finalidades de la enseñanza de las ciencias. Buenos Aires: Colihue, 1997.

FURIÓ, C. et al. Finalidades de la enseñanza de las ciencias en la secundaria obligatoria: ¿alfabetización científica o preparación propedéutica? Enseñanza de las Ciencias, Barcelona, v. 19, n. 3, p. 365-376, 2001.

GALLEGO TORRES, P. Imagen popular de la ciencia transmitida por los cómics. Revista Eureka sobre Enseñanza y Divulgación de las Ciencias, Cadiz, v. 4, n. 1, p. 141-151, 2007. 
Las imágenes inadecuadas de ciencia y de científico...

GALLEGOS, L.; BONILLA, M. Las concepciones sobre la naturaleza de la ciencia y la transformación de la práctica docente. Enseñanza de las Ciencias, Barcelona, p.106-112, 2009. (Número Extra: VIII Congreso Internacional sobre Investigación en Didáctica de las Ciencias).

GÓMEZ, S. et al. Escuelas con poblaciones en riesgo social: proyecto de intervención e investigación en el área de ciencias naturales. In: JORNADAS NACIONALES, 6. Y CONGRESO INTERNACIONAL DE ENSEÑANZA DE LA BIOLOGÍA, 1., 2004, Buenos Aires. Buenos Aires: Asociación de Docentes de Ciencias Biológicas de la Argentina, 2004.

HAYNES, R. From alchemy to artificial intelligence: stereotypes of the scientist in Western literature. Public Understanding of Science, London, v. 12, n. 3, p. 243-243, 2003.

HODSON, D. Science fiction: the continuing misrepresentation of science in the school curriculum. Pedagogy, Culture \& Society, Abingdon, v. 6, n. 2, p. 191-216, 1998.

HUGO, D.; ADÚRIZ-BRAVO, A. Algunos elementos teóricos para la investigación del conocimiento profesional del profesorado de ciencias naturales acerca de la naturaleza de la ciencia. In: ADÚRIZBRAVO, P. G.; BADILLO, E. (Ed.). Actualización en didáctica de las ciencias naturales y las matemáticas. Bogotá: Magisterio, 2003. p. 23-34.

JONES, M. G.; HOWE, A.; RUA, M. J. Gender differences in students' experiences, interests, and attitudes toward science and scientists. Science Education, Hoboken, v. 84, n. 2, p. 180-192, 2000.

LEDERMAN, N. Research on nature of science: reflections on the past, anticipations of the future. Asia-Pacific Forum of Science Learning and Teaching, Hong Kong, v. 7, n. 1, p. 1-11, 2006.

LESLIE-PELECKY, D. L.; BUCK, G. A. Y A.; ZABAWA, A. Broadening middle school students' images of science and scientists. Journal of Materials Education, Denton, v. 27, n. 3-6, p. 173-178, 2005.

LOSH, S.; WILKE, R.; POP, M. Some methodological issues with "Draw a Scientist Tests" among young children. International Journal of Science Education, Abingdon, v. 30, n. 6, p. 773-792, 2008.

MANASSERO MAS, M.; VÁZQUEZ ALONSO, A. Actitudes de estudiantes y profesorado sobre las características de los científicos. Enseñanza de las Ciencias, Barcelona, v. 19, n. 2, p. 255-268, 2001.

MANZOLI, F. et al. Children' s perceptions of science and scientists: a case study based on drawings and story-telling. In: INTERNATIONAL CONFERENCE ON PUBLIC COMMUNICATION OF SCIENCE AND TECHNOLOGY, 9., 2006, Seul.

MEAD, M.; METRAUX, R. Image of the scientist among high-school students. Science, Washington, v. 126, n. 3270, p. 384-390, 1957.

MORA, M. La teoría de las representaciones sociales de Serge Moscovici. Athenea Digital, Barcelona, v. 2, 2002. Disponible en: < http://atheneadigital.net/article/view/55>. Visitado el: 23 oct. 2012.

NEWTON, L. D.; NEWTON, D. P. Primary children's conceptions of science and the scientist: Is the impact of a National Curriculum breaking down the stereotype? International Journal of Science Education, Abingdon, v. 20, n. 9, p. 137-1149, 1998.

PUJALTE, A.; PORRO, S. Concepciones y actitudes del profesorado de ciencias acerca de la ciencia y la enseñanza y su relación con el logro de una educación científica para todas y todos. Enseñanza de las Ciencias, Barcelona, p. 181-184, 2009. (Número Extra VIII Congreso Internacional sobre Investigación en Didáctica de las Ciencias). 
PUJALTE, A. et al. ¿Qué nos imaginamos al pensar en la gente que se dedica a la ciencia?: implicaciones para una educación científica escolar de calidad para todas y todos. In: AVANCES EN EDUCACIÓN EN CIENCIA Y TECNOLOGÍA: ENFOQUES Y ESTRATEGIAS, 2011, San Fernando del Valle de Catamarca.

REIS, P.; GALVÃO, C. O diagnóstico de concepções sobre os cientistas através da análise e discussão de histórias de ficção científica redigidas pelos alunos. Revista Electrónica de Enseñanza de las Ciencias, Vigo, v. 5, n. 2, p. 213-234, 2006.

SJØBERG, S. Science and scientists: the SAS-study: cross-cultural evidence and perspectives on pupils' interests, experiences and perceptions : background, development and selected results. Oslo: Department of Teacher Education and School Development, University of Oslo: Unipub, 2000. (Acta didactica series, $1 / 2000)$.

STEELE, C. M. A threat in the air: how stereotypes shape intellectual identity and performance. The American Psychologist, Washington, v. 52, n. 6, p. 613-613, 1997.

STEKOLSCHIK, G. El científico, según la mirada de los niños, La Nación, Buenos Aires, 04 Mayo 2008. Disponible en: < http://www.lanacion.com.ar/nota.asp?nota_id=1009478>. Visitado el: 23 oct. 2012.

TSAI, C. C. Nested epistemologies: science teachers' beliefs of teaching, learning and science.

International Journal of Science Education, Abingdon, v. 24, n. 8, p. 771-783, 2002.

. Teachers' scientific epistemological views: the coherence with instruction and students' views.

Science Education, Hoboken, v. 91, n. 2, p. 222-243, 2007.

VÍLCHEZ-GONZÁLEZ, J. M.; PALACIOS, F. Image of science in cartoons and its relationship with the image in comics. Physics Education, Bristol, v. 41, n. 3, p. 240-249, 2006.

WEINGART, P. Chemists and their craft in fiction film: the public image of chemistry. Hyle: international journal for philosophy of chemistry, Karlsruhe, v. 12, n. 1, p. 31-44, 2007.

WYER, M. Intending to stay: images of scientists, attitudes toward women, and gender as influences on persistence among science and engineering majors. Journal of Women and Minorities in

Science and Engineering, Redding, v. 9, n. 1, p. 1-16, 2003.

Artigo recebido em 26/09/12. Aceito em 20/02/13. 